\title{
Introduction of plasmids into gastric cancer cells by endoscopic ultrasound
}

\author{
MINORU TOMIZAWA ${ }^{1}$, FUMINOBU SHINOZAKI ${ }^{2}$, YASUFUMI MOTOYOSHI ${ }^{3}$, \\ TAKAO SUGIYAMA $^{4}$, SHIGENORI YAMAMOTO ${ }^{5}$ and NAOKI ISHIGE ${ }^{6}$ \\ Departments of ${ }^{1}$ Gastroenterology, ${ }^{2}$ Radiology, ${ }^{3}$ Neurology, ${ }^{4}$ Rheumatology, ${ }^{5}$ Pediatrics and ${ }^{6}$ Neurosurgery, \\ National Hospital Organization, Shimoshizu Hospital, Yotsukaido, Chiba 284-0003, Japan
}

Received May 10, 2015; Accepted September 6, 2016

DOI: $10.3892 / \mathrm{ol} .2017 .5836$

\begin{abstract}
Short hairpin RNA of frizzled-2 (shRNA-Fz2) suppresses the cell proliferation of gastric cancer cells. Endoscopic ultrasound (EUS) is considered a suitable method for the introduction of therapeutic plasmids into cells, since the device enables the access and real-time monitoring of gastric cancer tissues. In the present study, plasmids were introduced into cells by sonoporation, as evidenced by the production of $\mathrm{H}_{2} \mathrm{O}_{2}$. The production of $\mathrm{H}_{2} \mathrm{O}_{2}$ was measured by absorbance of a potassium-starch solution irradiated with EUS. Luciferase activity was analyzed in the cells irradiated with EUS after the addition of a pMetLuc2-control in the media, and cell proliferation was analyzed using a 3-(4,5-dimethylthiazol-2-yl)-5(3-carboxymethoxyphenyl)-2-(4-sulfophenyl)-2H-tetrazolium inner salt assay after irradiation with EUS following the addition of shRNA-Fz2. Absorbance levels corresponding to free radical levels were found to be higher in the cells irradiated with EUS. Luciferase activities were found to be significantly higher in the transfected cells (plasmid with Lipofectamine LTX) than in untreated cells and were furthermore found to be higher in MKN45 cells irradiated for 0.5 min than in cells not subjected to irradiation. Luciferase activity was also found to be higher in MKN74 cells irradiated for 2 min than in cells that were not irradiated. Although the cell proliferation of the MKN45 cells tended to be suppressed by irradiation with EUS, this was non-significant suppression, while the cell proliferation of MKN74 cells was found to be suppressed by irradiation with $12 \mathrm{MHz}$ for $2 \mathrm{~min}(\mathrm{P}<0.05)$. In conclusion, plasmids were introduced into cultured gastric cancer cells by irradiation with EUS due to sonoporation, as evidenced by the production of $\mathrm{H}_{2} \mathrm{O}_{2}$; however, the efficiency of the plasmid
\end{abstract}

Correspondence to: Dr Minoru Tomizawa, Department of Gastroenterology, National Hospital Organization, Shimoshizu Hospital, 934-5 Shikawatashi, Yotsukaido, Chiba 284-0003, Japan E-mail: nihminor-cib@umin.ac.jp

Key words: frizzled-2, short hairpin RNA, sonoporation, free radical, hydrogen peroxide, potassium iodide starch method, MTS assay introduction was low compared with a traditional transfection approach.

\section{Introduction}

Frizzled (Fz)-2 is a receptor of the Wnt signaling pathway $(1,2)$. Fz-2 is expressed in gastric cancer tissues, and the proliferation and motility of gastric cancer cells have been shown to be suppressed by the short-hairpin RNA of Fz-2 (shRNA-Fz2) (3). The introduction of shRNA-Fz2 is therefore considered a novel target of molecular therapy, and methods of introducing shRNA-Fz2 must therefore be developed.

Sonoporation is a method of gene transfer to cells using ultrasonography (US) (4-7). Microbubbles grow and collapse when irradiated with US $(8,9)$, and during sonoporation, $\mathrm{H}_{2} \mathrm{O}_{2}$ is produced in the medium (10). This free radical production can be measured using the starch-iodide method (11), by which free radicals are quantified when combined with potassium iodide (KI) (12).

When microbubbles collapse, the cell membrane is perturbed and a pore is formed, allowing genetic material to enter the cell via the pore $(13,14)$. Diagnostic US is used to introduce plasmids and short interfering RNA into cultured cells in this manner (15-17). Diagnostic US may be a suitable means of introducing shRNA-Fz2 into gastric cancer tissues. It is occasionally difficult to visualize gastric cancer with extracorporeal US (18) and it would be challenging to introduce therapeutic genes into cancer tissues when the tissues are not adequately visualized with US. Endoscopy would thus be the ideal way in which to introduce genetic material to gastric cancer tissues, since the device also enables the visualization and monitoring of the cancer.

Endoscopic US (EUS) is indispensable for the diagnosis and staging of gastric cancer (19). EUS uses an endoscopic device fitted with US equipment on its tip and the probe is equipped with a balloon that is filled with water for the examination. The balloon is placed in direct contact with the area under investigation and the water in the balloon thus mediates the US between the probe and the area of examination.

In the present study, cultured gastric cancer cells were irradiated with EUS in an attempt to introduce shRNA-Fz2 into the cells. 


\section{Materials and methods}

Cell culture. The GC cell lines, MKN45 and MKN74, were purchased from RIKEN Cell Bank (Tsukuba, Japan). The cells were cultured in Roswell Park Memorial Institute (RPMI)-1640 medium (Sigma-Aldrich; Merck Millipore, Darmstadt, Germany) supplemented with $10 \%$ fetal bovine serum (FBS; Life Technologies; Thermo Fisher Scientific, Inc., Waltham, MA, USA). The cell lines were cultured with $5 \%$ carbon dioxide at $37^{\circ} \mathrm{C}$ in a humidified chamber. At $24 \mathrm{~h}$ prior to the experiments, the cells were split into 96-well fluoroimmunoassay (FIA) black plates (Greiner Bio-One, Frickenhausen, Germany), the bottoms of which consist of a transparent polystyrene film of $190 \pm 19 \mu \mathrm{m}$ in thickness. For irradiation with EUS or for transfection experiments, the cells were trypsinized, harvested, seeded into 96-well FIA black plates $\left(1,000\right.$ cells/well) and incubated for $24 \mathrm{~h}$ at $37^{\circ} \mathrm{C}$ in RPMI-1640 supplemented with 10\% FBS. The following day, the cultured cells were subjected to irradiation or transfection.

Irradiation with EUS. The probe of the EUS device (GF-UCT260; Olympus, Tokyo, Japan) was covered with a balloon (Fig. 1A). The balloon was filled and enlarged with water to mediate the US (Fig. 1B). The balloon was then attached to the bottom of a 96-well FIA black plate, after which ultrasound was irradiated through the water to the bottom of the wells (Fig. 1C). Plasmid (100 ng) was added to the cells in $25 \mu$ l Opti-Minimal Essential Medium (Opti-MEM) Reduced Serum Media (Life Technologies; Thermo Fisher Scientific, Inc.), after which the cell-plasmid mixture was irradiated with EUS for 0.5 or $2 \mathrm{~min}$. After the irradiation, $25 \mu 1 \mathrm{RPMI}$ supplemented with $10 \%$ FBS was added to the cells. The irradiation field was monitored in real-time using the display of the EUS device (Fig. 1D).

Transfection. Cultured cells were transfected with $100 \mathrm{ng}$ plasmid using Lipofectamine LTX (Life Technologies; Thermo Fisher Scientific, Inc.) according to the manufacturer's instructions: Plasmids for transfection were added to the cells in $25 \mu \mathrm{l}$ Opti-MEM and allowed to complex with Lipofectamine LTX. The complex was mixed with $25 \mu 1$ RPMI-1640 supplemented with $10 \%$ FBS.

Quantification of $\mathrm{H}_{2} \mathrm{O}_{2}$ generation. The generation of $\mathrm{H}_{2} \mathrm{O}_{2}$ was quantified using the starch-iodide method (20). Potassium iodide $(100 \mu \mathrm{l} ; 0.05 \mathrm{M})$ and starch $(5 \mathrm{mg} / \mathrm{ml})$ were placed into each well of the 96-well FIA black plates. Any $\mathrm{H}_{2} \mathrm{O}_{2}$ produced by irradiation with EUS oxidizes $\mathrm{I}^{-}$into $\mathrm{I}_{2}$, which then reacts with starch to form a purple-colored complex. The resulting absorbance at $490 \mathrm{~nm}$ was analyzed using an iMark Microplate Absorbance Reader (Bio-Rad Laboratories, Inc., Hercules, CA, USA).

Luciferase assay. Luciferase assays were performed with pMetLuc2-control (Promega Corporation, Madison, WI, USA). In the luciferase assay, Metridia luciferase, the expression of which is driven by a cytomegalovirus immediate early promoter, is secreted into the medium. The medium from the gastric cancer cells was collected $48 \mathrm{~h}$ after irradiation

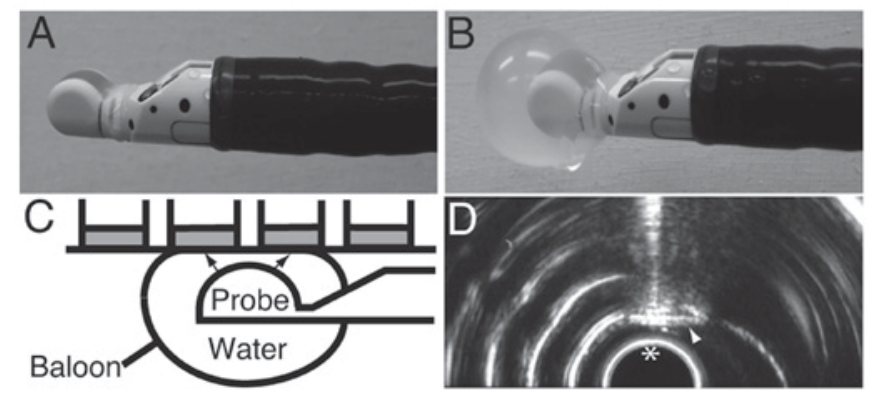

Figure 1. Irradiation with EUS. A balloon attached to the (A) EUS probe is (B) dilatated with water. (C) Fluid (gray rectangles) is irradiated with EUS (arrow) via the fluid-filled balloon through the bottom of the 96-well plate. (D) Image showing the irradiation field (the arrowhead indicates the air above the fluid in the well of the 96-well plate; the asterisk indicates the probe of the endoscopic ultrasound).

with EUS or transfection, and enzyme activity was assayed using a Ready-To-Glow Secreted Luciferase Reporter assay (Clontech Laboratories, Inc., Mountain View, CA, USA) and a Gene Light (GL-200A) luminometer (Microtech Co., Ltd., Funabashi, Japan). To monitor transfection efficiency, $10 \mathrm{ng}$ of the pSEAP2 control vector (Clontech Laboratories, Inc.) was used in each well of the 96-well FIA black plates and transcriptional activity was measured using a secreted embryonic alkaline phosphatase (SEAP) chemiluminescence kit (Clontech Laboratories, Inc.) and the Gene Light luminometer according to the manufacturer's instructions. Luciferase activity was calculated as the Metridia luciferase activity divided by the SEAP activity.

Cell proliferation analysis. After $72 \mathrm{~h}$ of incubation at $37^{\circ} \mathrm{C}$ following irradiation withEUS or transfection with shRNA-Fz2, the cells were subjected to 3-(4,5-dimethylthiazol-2-yl)-5-(3carboxymethoxyphenyl)-2-(4-sulfophenyl)-2H-tetrazolium inner salt (MTS) assays according to the manufacturer's instructions (Promega Corporation). The assay is based on the bio-reduction of MTS by live cells into a colored formazan product that can be quantified by absorbance at $490 \mathrm{~nm}$. Absorbance was analyzed at a wavelength of $490 \mathrm{~nm}$ using an iMark Microplate Absorbance Reader (Bio-Rad Laboratories, Inc.).

Statistical analysis. Absorbance was assessed by a one-factor analysis of variance using JMP5.0 J software (SAS Institute, Cary, NC, USA). $\mathrm{P}<0.05$ was considered to indicate a statistically significant difference.

\section{Results}

To assess whether free radicals were produced by the EUS performed in this study, absorbance was measured in cell cultures after irradiation with EUS or transfection (Fig. 2). Absorbance levels were found to be higher in wells irradiated for $0.5 \mathrm{~min}$ at $5 \mathrm{MHz}(\mathrm{P}<0.05)$ and for $2 \mathrm{~min}$ at $12 \mathrm{MHz}$ $(\mathrm{P}<0.05)$ compared with absorbance levels in the control cells, suggesting that free radicals may be produced by irradiation with EUS. Although the absorbance levels were higher for $0.5 \mathrm{~min}$ at $5 \mathrm{MHz}$ and for $2 \mathrm{~min}$ at $12 \mathrm{MHz}$, the differences were not significant for $2 \mathrm{~min}$ at $5 \mathrm{MHz}(\mathrm{P}=0.0820)$ or for $0.5 \mathrm{~min}$ at $12 \mathrm{MHz}(\mathrm{P}=0.1220)$. 


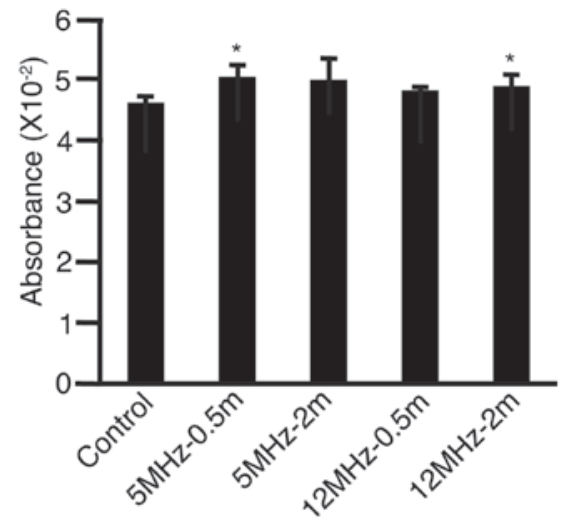

Figure 2. Absorbance of mixtures of KI and starch following irradiation with EUS. Absorbance readings of mixtures of KI and starch in 96-well plates were analyzed after irradiation with EUS. ${ }^{*} \mathrm{P}<0.05$ compared with the control group ( $\mathrm{n}=4)$. Control, no irradiation; $5 \mathrm{MHz}-0.5 \mathrm{~m}$, irradiated with $5 \mathrm{MHz}$ for $0.5 \mathrm{~min} ; 5 \mathrm{MHz}-2 \mathrm{~m}$, irradiated with $5 \mathrm{MHz}$ for $2 \mathrm{~min} ; 12 \mathrm{MHz}-0.5 \mathrm{~m}$, irradiated with $12 \mathrm{MHz}$ for $0.5 \mathrm{~min} ; 12 \mathrm{MHz}-2 \mathrm{~m}$ : irradiated with $12 \mathrm{MHz}$ for 2 min; EUS, endoscopic ultrasound; KI, potassium iodide.

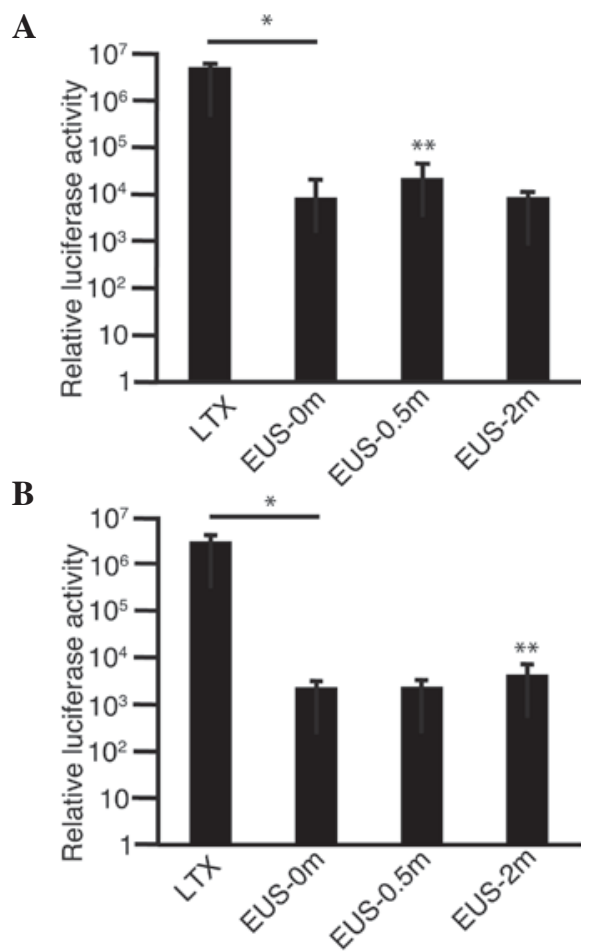

Figure 3. Luciferase assay following irradiation with EUS. (A) MKN45 and (B) MKN74 gastric cancer cells were cultured in the wells of 96-well plates. Reporter plasmids were added into the culture media, cells were irradiated with EUS at $5 \mathrm{MHz}$ and the luciferase assay was performed. The $\mathrm{y}$-axis scale is logarithmic. " $\mathrm{P}<0.05$ compared with the LTX group $(\mathrm{n}=3)$; ${ }^{* *} \mathrm{P}<0.05$ compared with the EUS-0m group. LTX, transfection with reagent (Lipofectamine LTX); EUS-0, no irradiation; EUS-0.5m, irradiation for 0.5 min with EUS; EUS- $2 \mathrm{~m}$, irradiation for $2 \mathrm{~min}$ with EUS. EUS, endoscopic ultrasound.

To confirm that plasmids were introduced into the gastric cancer cells, MKN45 (Fig. 3A) or MKN74 (Fig. 3B) cells were combined with pMetLuc2-control in the medium, irradiated with EUS and subjected to a luciferase assay. Luciferase activity was found to be significantly higher in transfected cells (LTX) than in the cells that were untreated
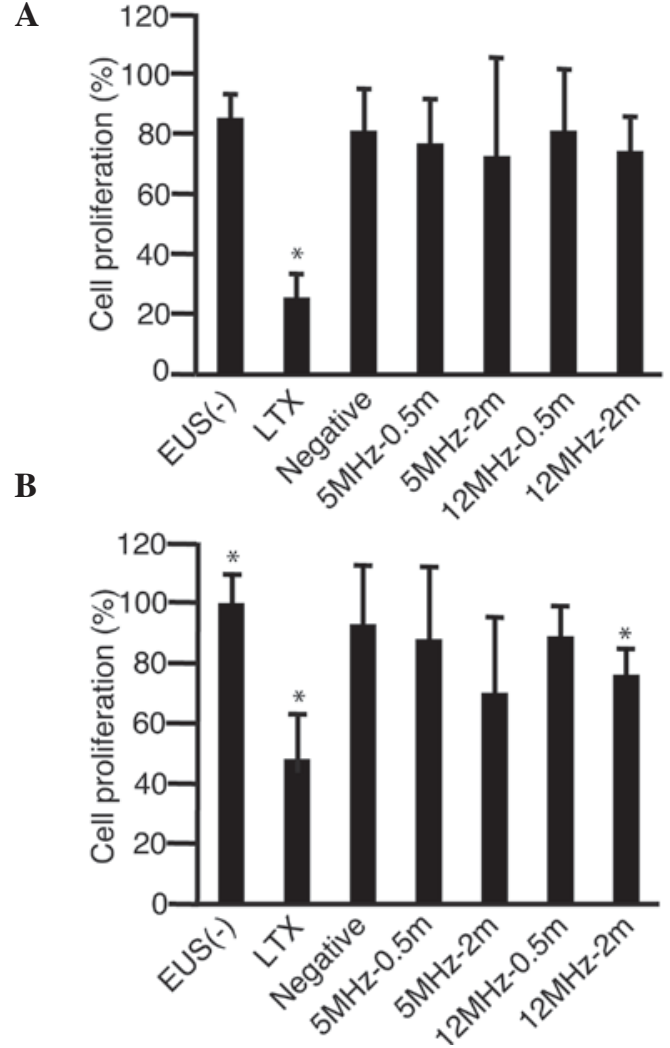

Figure 4. Cell proliferation assay following irradiation with EUS shRNA-Fz2 was added into the medium of the (A) MKN45 and (B) MKN74 gastric cancer cells. The 3-(4,5-dimethylthiazol-2-yl)-5-(3carboxymethoxyphenyl)-2-(4-sulfophenyl)-2H-tetrazolium inner salt assay was then performed to analyze cell proliferation $72 \mathrm{~h}$ after irradiation with EUS. * $\mathrm{P}<0.05$ compared with the EUS(-) group ( $\mathrm{n}=3)$. EUS(-), addition of shRNA-Fz2 without irradiation with EUS; LTX, shRNA-Fz2 transfection with Lipofectamine LTX; Negative, addition of negative control of short-hairpin RNA; 5MH-0.5m, addition of shRNA-Fz2 and 0.5 min irradiation with $5 \mathrm{MHz} ; 5 \mathrm{MHz}-2 \mathrm{~m}$, addition of shRNA-Fz2 and 2 min irradiation with $5 \mathrm{MHz} ; 12 \mathrm{MHz}-0.5 \mathrm{~m}$, addition of shRNA-Fz2 and $0.5 \mathrm{~min}$ irradiation with $12 \mathrm{MHz} ; 12 \mathrm{MHz}-2 \mathrm{~m}$, addition of shRNA-Fz2 and 2 min irradiation with $12 \mathrm{MHz}$; shRNA-Fz2, short-hairpin RNA of frizzled-2; EUS, endoscopic ultrasound.

(EUS-0m) $(\mathrm{P}<0.0001)$. Luciferase activity was higher in the MKN45 cells subjected to 0.5 min irradiation (EUS-0.5m) than in the untreated cells (EUS-0m) $(\mathrm{P}=0.0329)$ (Fig. 3A). In the MKN74 cells subjected to 2 min irradiation (EUS-2m), luciferase activity was found to be higher than that in the untreated cells (EUS-0m) ( $\mathrm{P}=0.0219)$ (Fig. 3B). These findings suggest that plasmids are indeed introduced into the cells by irradiation with EUS even though the efficiencies were lower than those achieved with the conventional transfection reagents.

To investigate whether shRNA-Fz2 suppressed the proliferation of MKN45 (Fig. 4A) and MKN74 (Fig. 4B) cells, the plasmids were added into the cell medium and the cells were then irradiated with EUS. Proliferation was found to be significantly suppressed by transfection (LTX) in the two cell lines. The proliferation of the MKN45 cells tended to be suppressed by irradiation with EUS, but without statistical significance (Fig. 4A). The proliferation of the MKN74 cells was found to be suppressed by irradiation with $12 \mathrm{MHz}$ for $2 \min (\mathrm{P}<0.0001)$ (Fig. 4B). 


\section{Discussion}

$\mathrm{H}_{2} \mathrm{O}_{2}$ is produced by irradiation with US. Okada et al irradiated water in $35-\mathrm{mm}$ dishes and measured $\mathrm{H}_{2} \mathrm{O}_{2}$ generation by the KI-starch method (11). In their experiments, absorbance values $(555 \mathrm{~nm})$ ranged from 0 to 0.35 . In the present experiments, absorbance values at $490 \mathrm{~nm}$ ranged from 0.045 to 0.055, suggesting that EUS caused sonoporation (21). The increases in absorbance that were reported in the present study are not as significant as those reported by Okada et al, leading us to speculate that $\mathrm{H}_{2} \mathrm{O}_{2}$ is produced at lower levels by irradiation with EUS than by devices designed specifically for irradiation experiments (11). The low levels of $\mathrm{H}_{2} \mathrm{O}_{2}$ production may also be a result of EUS being biologically safe and producing only low levels of free radicals (22). The standing wave effects may also have enhanced the production of $\mathrm{H}_{2} \mathrm{O}_{2}$ in previous studies (23): Kinoshita and Hynynen set a water chamber on the surface of samples to eliminate the standing wave effects (24).

Therapeutic genes have been introduced into gastric cancer cells to suppress their proliferation $(3,25)$, and in the present study, EUS was assessed as a method for the introduction of such therapeutic genes into cells. A luciferase assay showed that the reporter plasmids were indeed introduced into the cells; however, the efficiency of the introduction of the genes by EUS was lower than that of introduction by transfection. Cell proliferation tended to be suppressed following irradiation by EUS after the addition of shRNA-Fz2, although with no statistical significance except for in MKN74 cells irradiated with $12 \mathrm{MHz}$ for $2 \mathrm{~min}$ (Fig. 4). Suppression of cell proliferation by shRNA-Fz2 was less than that achieved by conventional transfection methods, possibly due to the lower efficiency of introduction with irradiation by EUS compared with transfection.

A limitation of this study is the fact that the efficiency of the EUS-mediated plasmid introduction was low compared with the efficiency of plasmid introduction by conventional transfection reagents. Microbubbles enhance sonoporation and increase introduction efficiencies of plasmids (26) and EUS was therefore expected to achieve more efficient introduction of shRNA-Fz2 than traditional methods. Further studies would thus include the application of microbubbles to the culture media of cells to increase the efficiency of shRNA-Fz2 introduction.

In conclusion, plasmids were successfully introduced into cultured gastric cancer cells by sonoporation achieved by irradiation with EUS, as evidenced by the production of $\mathrm{H}_{2} \mathrm{O}_{2}$; however, the efficiency of this plasmid introduction method was found to be low compared with traditional transfection methods.

\section{References}

1. Tanaka SS, Kojima Y, Yamaguchi YL, Nishinakamura R and Tam PP: Impact of WNT signaling on tissue lineage differentiation in the early mouse embryo. Dev Growth Differ 53: 843-856, 2011.

2. MacDonald BT, Tamai K and He X: Wnt/beta-catenin signaling: Components, mechanisms and diseases. Dev Cell 17: 9-26, 2009

3. Tomizawa M, Shinozaki F, Motoyoshi Y, Sugiyama T, Yamamoto $\mathrm{S}$ and Ishige N: Gastric cancer cell proliferation is suppressed by frizzled-2 short hairpin RNA. Int J Oncol 46: 1018-1024, 2015.
4. Feril LB Jr and Tachibana K: Use of ultrasound in drug delivery systems: Emphasis on experimental methodology and mechanisms. Int J Hyperthermia 28: 282-289, 2012.

5. Fechheimer M, Boylan JF, Parker S, Sisken JE, Patel GL and Zimmer SG: Transfection of mammalian cells with plasmid DNA by scrape loading and sonication loading. Proc Natl Acad Sci USA 84: 8463-8467, 1987.

6. Kim HJ, Greenleaf JF, Kinnick RR, Bronk JT and Bolander ME: Ultrasound-mediated transfection of mammalian cells. Hum Gene Ther 7: 1339-1346, 1996.

7. Tomizawa M, Ebara M, Saisho H, Sakiyama S and Tagawa M: Irradiation with ultrasound of low output intensity increased chemosensitivity of subcutaneous solid tumors to an anti-cancer agent. Cancer Lett 173: 31-35, 2001.

8. O'Brien WD Jr: Ultrasound-biophysics mechanisms. Prog Biophys Mol Biol 93: 212-255, 2007.

9. Newman CM and Bettinger T: Gene therapy progress and prospects: Ultrasound for gene transfer. Gene Ther 14: 465-475, 2007.

10. Wang P, Li Y, Wang X, Guo L, Su X and Liu Q: Membrane damage effect of continuous wave ultrasound on K562 human leukemia cells. J Ultrasound Med 31: 1977-1986, 2012.

11. Okada K, Kudo N, Hassan MA, Kondo T and Yamamoto K: Threshold curves obtained under various gaseous conditions for free radical generation by burst ultrasound-Effects of dissolved gas, microbubbles and gas transport from the air. Ultrason Sonochem 16: 512-518, 2009.

12. Ebrahiminia A, Mokhtari-Dizaji M and Toliyat T: Correlation between iodide dosimetry and terephthalic acid dosimetry to evaluate the reactive radical production due to the acoustic cavitation activity. Ultrason Sonochem 20: 366-372, 2013.

13. Qiu Y, Zhang C, Tu J and Zhang D: Microbubble-induced sonoporation involved in ultrasound-mediated DNA transfection in vitro at low acoustic pressures. J Biomech 45: 1339-1345, 2012.

14. Kudo N, Okada K and Yamamoto K: Sonoporation by single-shot pulsed ultrasound with microbubbles adjacent to cells. Biophys J 96: 4866-4876, 2009.

15. Miller DL and Quddus J: Sonoporation of monolayer cells by diagnostic ultrasound activation of contrast-agent gas bodies. Ultrasound Med Biol 26: 661-667, 2000.

16. Tomizawa M, Shinozaki F, Sugiyama T, Yamamoto S, Sueishi M and Yoshida T: Plasmid DNA introduced into cultured cells with diagnostic ultrasound. Oncol Rep 27: 1360-1364, 2012.

17. Tomizawa M, Shinozaki F, Sugiyama T, Yamamoto S, Sueishi M and Yoshida T: Short interference RNA introduced into cultured cells with diagnostic ultrasound. Oncol Rep 27: 65-68, 2012.

18. Tomizawa M, Shinozaki F, Hasegawa R, Fugo K, Shirai Y, Ichiki N, Sugiyama T, Yamamoto S, Sueishi M and Yoshida T: Screening ultrasonography is useful for the diagnosis of gastric and colorectal cancer. Hepatogastroenterology 60: 517-521, 2013.

19. El Abiad R and Gerke H: Gastric cancer: Endoscopic diagnosis and staging. Surg Oncol Clin N Am 21: 1-19, 2012.

20. Kondo T and Yoshii G: Effect of intensity of $1.2 \mathrm{MHz}$ ultrasound on change in DNA synthesis of irradiated mouse L cells. Ultrasound Med Biol 11: 113-119, 1985.

21. Tomizawa M, Shinozaki F, Motoyoshi Y, Sugiyama T, Yamamoto $\mathrm{S}$ and Sueishi M: Sonoporation: Gene transfer using ultrasound. World J Methodol 3: 39-44, 2013.

22. WFUMB Symposium on safety and standardisation in medical ultrasound. Issues and recommendations regarding thermal mechanisms for biological effects of ultrasound. Hornbaek, denmark, 30 August-1. Ultrasound Med Biol 18: 731-810, 1992.

23. Hassan MA, Buldakov MA, Ogawa R, Zhao QL, Furusawa Y, Kudo N, Kondo T and Riesz P: Modulation control over ultrasound-mediated gene delivery: Evaluating the importance of standing waves. J Control Release 141: 70-76, 2010.

24. Kinoshita M and Hynynen K: Key factors that affect sonoporation efficiency in in vitro settings: The importance of standing wave in sonoporation. Biochem Biophys Res Commun 359: 860-865, 2007.

25. Wang TS, Ding QQ, Guo RH, Shen H, Sun J, Lu KH, You SH, Ge HM, Shu YQ and Liu P: Expression of livin in gastric cancer and induction of apoptosis in SGC-7901 cells by shRNA-mediated silencing of livin gene. Biomed Pharmacother 64: 333-338, 2010.

26. Yamaguchi K, Feril LB Jr, Tachibana K, Takahashi A, Matsuo M, Endo H, Harada Y and Nakayama J: Ultrasound-mediated interferon $\beta$ gene transfection inhibits growth of malignant melanoma. Biochem Biophys Res Commun 411: 137-142, 2011. 